\title{
Application of Three Order Cumulants in Fault Diagnosis
}

\author{
Wenbing Wu \\ Department of Mathematics and Computer Science, Nanchang Normal University, Nanchang, China \\ Email: wwbysq@fjnu.edu.cn
}

How to cite this paper: $\mathrm{Wu}, \mathrm{W} . \mathrm{B} .(2018)$ Application of Three Order Cumulants in Fault Diagnosis. International Journal of Modern Nonlinear Theory and Application, 7, 97-105.

https://doi.org/10.4236/ijmnta.2018.74008

Received: November 11, 2018

Accepted: December 21, 2018

Published: December 24, 2018

Copyright $\odot 2018$ by author and Scientific Research Publishing Inc. This work is licensed under the Creative Commons Attribution International License (CC BY 4.0).

http://creativecommons.org/licenses/by/4.0/

\section{(c) (i) Open Access}

\begin{abstract}
The three-order cumulants' complex forms of different definitions include different coupling information of signals, and the information can be used to diagnose fault. In the experiment of pressure reducing valve's fault diagnosis, through these different coupling information, the features of fault signals and normal signals were extracted by wavelet in different directions, then these features were inputted to diagnose the fault. The experiment shows that this method can achieve a satisfactory result.
\end{abstract}

\section{Keywords}

Cumulants, Coupling, Wavelet, Fault Diagnosis

\section{Introduction}

High-order cumulants can automatically suppress the influence of Gaussian background noise (colored or white), establish a non-Gaussian signal model under Gaussian noise, and extract non-Gaussian signals (including harmonic signals) in Gaussian noise [1]. Because of this, the statistic of high-order cumulants has received increasing attention and has become a very useful tool in signal processing. The bispectrum derived from the third-order cumulant contains asymmetric and nonlinear information of the signal, which can be used to describe nonlinear phase coupling, especially quadratic phase coupling, which has been widely used in fault diagnosis [2] [3] [4].

Stronach et al. conducted in-depth research on high-order spectra and applied them to various mechanical fault diagnosis [5] [6]; WR Raghuveer et al. and domestic scholar Wang Shuxun et al. conducted in-depth research on the coupling properties of higher-order cumulants, clearly pointed out the coupling characteristics of different definitions of various complex higher-order cumu- 
lants [7] [8] [9]. The author of this paper has studied high-order cumulants for many years, and has published many theses on this topic. Wavelet analysis is a powerful tool for signal analysis with its good time-frequency characteristics, especially for the analysis of nonlinear and non-stationary signals. Compared with the Fourier transform, wavelet analysis can analyze the short-term high-frequency components of the signal with sufficient time resolution, and estimate the low-frequency of the signal at a good frequency resolution. Artificial neural network is an adaptive nonlinear dynamic information processing system. It can change the network weight through sample learning training, so that the actual output and expected output meet the specified error requirements. Therefore, the neural network model can be used as a fault classifier for fault diagnosis. Since the actual mechanical vibration signal has nonlinear and non-stationary characteristics, and the wavelet transform can effectively process such signals, this paper uses the two-dimensional wavelet for feature extraction according to the different coupling characteristics of the pressure-reducing valve vibration signal, and then extracts the feature value input. Least Square Support Vector Machine (LS-SVM) for fault diagnosis has achieved good results.

\section{Higher Order Cumulant}

Let $\{x(n)\}$ be a zero-mean $k$-order stationary stochastic process, then the $k$-order cumulant of the process is defined as the k-order joint cumulant of the random variable.

$$
c_{k x}\left(\tau_{1}, \tau_{2}, \cdots, \tau_{k-1}\right)=\operatorname{cum}\left\{x(n), x\left(n+\tau_{1}\right), x\left(n+\tau_{2}\right), \cdots, x\left(n+\tau_{k-1}\right)\right\}
$$

The $k$-order moment of the process $m_{k x}\left(\tau_{1}, \tau_{2}, \cdots, \tau_{k-1}\right)$ is defined as the $k$-order joint moment of the random variable $\left\{x(n), x\left(n+\tau_{1}\right), \cdots, x\left(n+\tau_{k-1}\right)\right\}$, which is

$$
m_{k x}\left(\tau_{1}, \tau_{2}, \cdots, \tau_{k-1}\right)=\operatorname{mom}\left\{x(n), x\left(n+\tau_{1}\right), \cdots, x\left(n+\tau_{k-1}\right)\right\}
$$

Here, $\operatorname{mom}()$ represents the joint moment, and the third-order cumulant is

$$
c_{3 x}\left(\tau_{1}, \tau_{2}\right)=E\left\{x(n) x\left(n+\tau_{1}\right) x\left(n+\tau_{2}\right)\right\}
$$

\section{Third-Order Cumulant Coupling Properties}

According to the literature [8], in Equation (3), let $x(n)$ be a complex signal and define it as follows:

Definition one:

$$
c_{3 x}\left(\tau_{1}, \tau_{2}\right)=E\left\{x(n) x\left(n+\tau_{1}\right) x\left(n+\tau_{2}\right)\right\}
$$

Definition two:

$$
c_{3 x}\left(\tau_{1}, \tau_{2}\right)=E\left\{x^{*}(n) x\left(n+\tau_{1}\right) x\left(n+\tau_{2}\right)\right\}
$$

Definition three:

$$
c_{3 x}\left(\tau_{1}, \tau_{2}\right)=E\left\{x^{*}(n) x\left(n+\tau_{1}\right) x^{*}\left(n+\tau_{2}\right)\right\}
$$


Among them, $x^{*}(n)$ is the conjugate complex number of $x(n)$. The complex signal in this paper is obtained by hilbert transform from the original signal collected. Reference [6] points out that, according to the definition one, signals in which quadratic phase coupling occurs can be represented in complex harmonic form (such as Equation (7), among them $\phi_{3}=\phi_{2}+\phi_{1}$, harmonic components $\omega_{3}$ are formed by harmonic components $\omega_{1}$ and $\omega_{2}$ by secondary phase coupling, and $\left.\omega_{3}=\omega_{1}+\omega_{2}\right)$ and its third-order cumulant $C_{1}$ is 0 , in the manner of defining two, its third-order cumulant $C_{2}$ is as shown in Equation (8), and its third-order cumulant $C_{3}$ is defined as Equation (9) shown.

$$
\begin{gathered}
x(n)=\sum_{i=1}^{3} A_{i} \exp \left(\omega_{i} n\right)+\phi_{i} \\
C_{2}=A_{1} A_{2} A_{3}\left[\exp \left[j\left(\omega_{1} \tau_{1}+\omega_{2} \tau_{2}\right)\right]+\exp \left[j\left(\omega_{2} \tau_{1}-\omega_{1} \tau_{2}\right)\right]\right] \\
C_{3}=A_{1} A_{2} A_{3}\left[\exp \left[j\left(\omega_{3} \tau_{1}-\omega_{1} \tau_{2}\right)\right]+\exp \left[j\left(\omega_{3} \tau_{1}-\omega_{2} \tau_{2}\right)\right]\right]
\end{gathered}
$$

It can be seen from Equation (8) that in the second way, its third-order cumulant contains only the harmonic signals $\omega_{1}$ and $\omega_{2}$ that participate in the coupling, and the third-order cumulant in the third way contains both harmonic signals that participate in coupling $\omega_{1}$ and $\omega_{2}$ and also contains the coupled signal.

\section{Two-Dimensional Wavelet Multi-Scale Decomposition}

Wavelet transform can be used for nonlinear, non-stationary mechanical vibration signals. It has the ability to characterize the local features of the signal in both time and frequency domains, it is a time-frequency localization analysis method in which the window size is fixed but its shape is variable, and both the time window and the frequency window are variable. The two-dimensional wavelet function is obtained by tensor product transformation through one-dimensional wavelet function.

\section{Data Collection}

The pressure reducing valve in this experiment is a pilot type pressure reducing valve. When the pressure reducing valve has foreign matter in and out of the oil port, the pressure is high or low, which will affect the normal operation of the pressure reducing valve, In order to obtain the operating signal of the pressure reducing valve in the fault state, the experiment conducted in this paper artificially sets the following faults: (Figure 1)

Add $\phi 3 \mathrm{~mm}$ core to the pressure inlet of the pressure reducing valve.

Through the experiment, the working condition of the pressure reducing valve can be approximated.

This paper uses LabVIEW software and PCI-6014 data acquisition card and an acceleration sensor to sequentially collect the vibration signal of the pressure reducing valve under normal and fault conditions. In each measurement, the oil pressure is divided into five pressure levels from $1 \mathrm{MPa}$ to $5 \mathrm{MPa}$. The sampling 


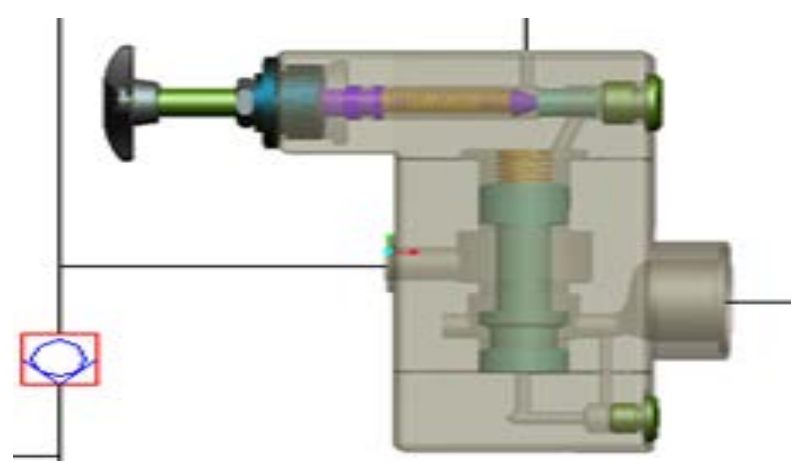

Figure 1. Reducing valve.

frequency is $250 \mathrm{~Hz}$, the reading frequency is $125 \mathrm{~Hz}$, and the sampling process time is about $2 \mathrm{~min}$. In this experiment, 18 groups of 36 sets of data were collected in the normal working state and the fault state of the pressure reducing valve. The number of data used in this experiment is 1536.

\section{Complex Third-Order Cumulant Map}

In this experiment, the 36 sets of data of the normal state and the fault state are first obtained, and the third-order cumulant of each set of data is obtained according to the above three definitions, in this paper, we select the complex third-order cumulant of the normal state and fault state data, let $-127 \leq \tau_{1}, \tau_{2} \leq 127$, and then when the oil pressure is $1 \mathrm{MPa}, 3 \mathrm{MPa}, 5 \mathrm{MPa}$, in the definition of the three modes, the third-order cumulant of the two states is taken as an absolute value, and each of the selected groups is shown in Figure 2 and Figure 3. In the figure, the $\mathrm{x}$ and $\mathrm{y}$ axes represent the number of data, the unit is one, and the vertical axis indicates the normalized amplitude, dimensionless. It can be seen from Figure 2 and Figure 3 that the peak distribution of third-order cumulant of the fault state is sparser than the normal state.

\section{Feature Extraction}

In the fault identification, if the third-order cumulant data obtained is directly input into the neural network, the calculation amount is too large, and the diagnosis result obtained by the experiment is not satisfactory. Since the various frequency information contained in the original signal must be reflected in its third-order cumulant, and wavelet decomposition can effectively extract these frequency information, therefore, this paper uses two-dimensional wavelet decomposition to extract features that the third-order cumulant of different coupling modes of each measured data, this article uses the db1 wavelet [8] [9]. through experimental comparison and analysis, after the 2D wavelet function decomposition of the original data, and the 6-level compression coefficient with the scale of 6 is extracted as the LSSVM input vector, and the best recognition effect is obtained, the compressed coefficient is a $4 * 4$ two-dimensional array. In this paper, the feature extraction is performed in the high frequency, Figure 4 

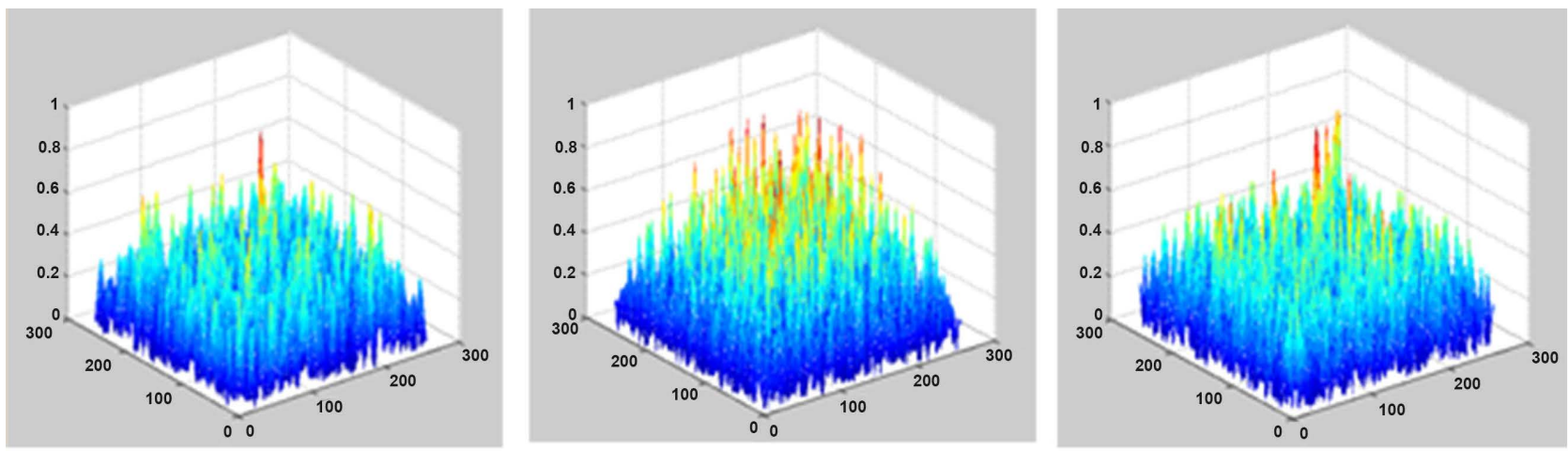

Figure 2. Complex three order cumulants of definition 3 in normal state.
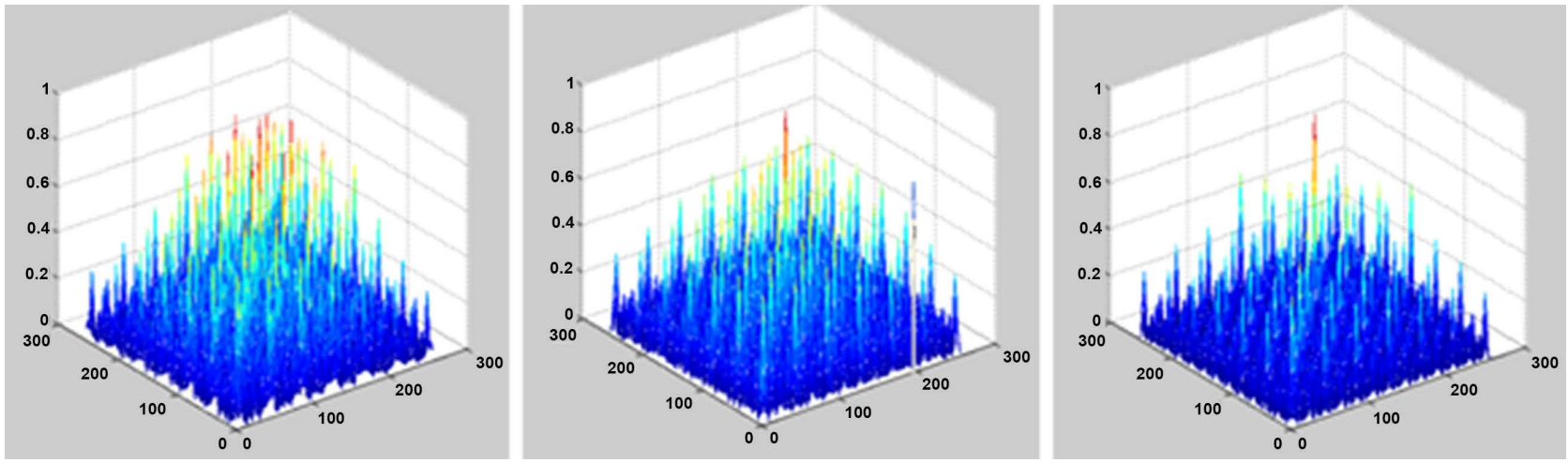

Figure 3. Complex three order cumulants of definition 3 in fault state.
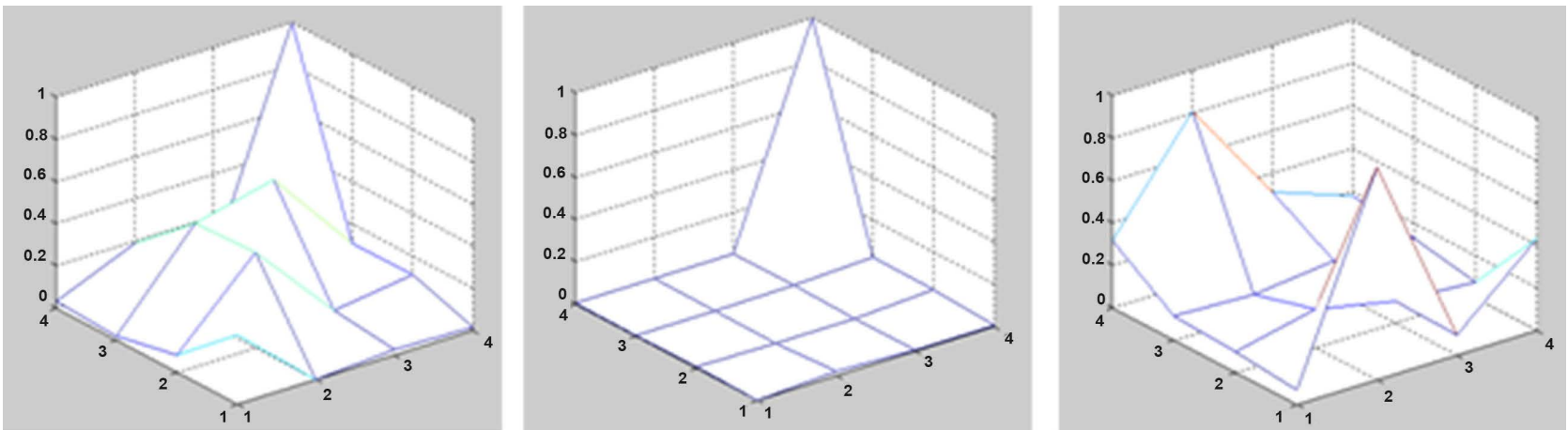

Figure 4. Wavelet coefficients of definition 3 in normal state.

and Figure 5 show the values after compression and modulo of the high-frequency, where the $\mathrm{x}$ and $\mathrm{y}$ axes represent the dimensions of the compressed two-dimensional matrix, and the $\mathrm{z}$-axis represents the extracted normalized eigenvalues, all of which are dimensionless. Table 1 shows the feature vectors obtained by compressing the normal state and fault state data in the high frequency in the definition three modes.

\section{Experimental Result}

Mechanical fault diagnosis includes three parts: signal acquisition, signal processing and fault mode classification. Fault mode classification is the core 

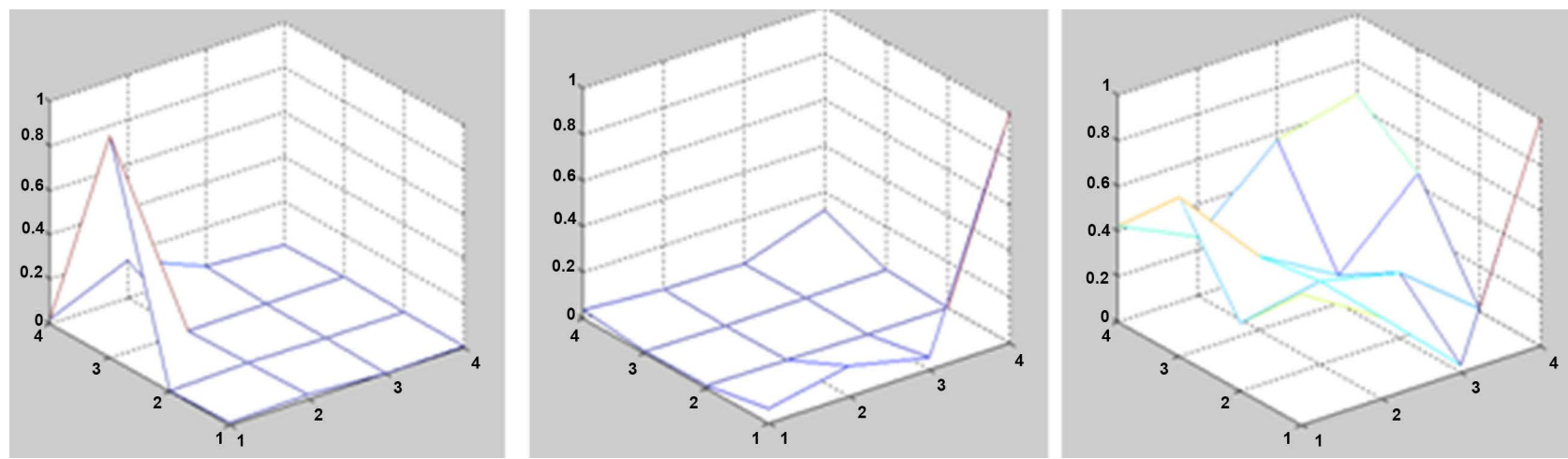

Figure 5. Wavelet coefficients of definition 3 in normal state.

Table 1. Complex three order cumulants' features extracted by 2-D wavelet in diagonal direction of definition 3.

\begin{tabular}{clllllllll}
\hline & 0.2303 & 0.3309 & 0.0777 & 06620 & 0.4379 & 0.5280 & 0.0139 & 0.3194 \\
$\begin{array}{c}\text { Normal } \\
\text { status }\end{array}$ & 0.3131 & 0.3222 & 0.3725 & 0.3740 & 0.3357 & 0.0323 & 0.3156 & 0.0246 \\
& 0.2426 & 0.4528 & 0.2681 & 0.4695 & 0.5328 & 0.2329 & 0.5135 & 0.5177 \\
& 0.3236 & 0.3258 & 0.3728 & 0.6658 & 0.4346 & 0.0298 & 0.3191 & 0.6284 \\
Fault & 0.1832 & 0.4721 & 0.5733 & 0.5671 & 0.0350 & 0.5306 & 0.0156 & 0.5230 \\
status & 03644 & 0.3464 & 0.2572 & 0.0669 & 0.5265 & 0.4311 & 0.3144 & 0.3231 \\
& & & & & & & &
\end{tabular}

content of diagnosis. Support Vector Machine (SVM) is proposed to solve the two-class classification problem and has rapidly developed into a powerful tool for classification problems. It has been successfully applied in many engineering fields, such as speech recognition, image classification and so on. LSSVM is an improved algorithm based on SVM method. The LSSVM method uses a least squares linear system as the loss function. Unlike the classic SVM, it has only equality constraints and no inequality constraints, which speeds up the calculation. In this paper, the same LSSVM is established according to the literature [10]. When training the LSSVM, the normal state and the fault state are respectively coded as 1 and -1 , since the normal data and the fault data are obtained under five different oil pressures respectively, the corresponding five groups of data are taken out in the normal data and the fault data of Table 1 respectively, and the set of least squares is established is used for training, after the training, the support vector machine is used for fault identification, the remaining 26 sets of data are simulated and identified by the trained network. As shown in Figure 6.

The figure shows the simulated state of 26 sets of data, in which the open circle indicates the preset state of the training data, for example, the first 13 sets of data are measured normal data, which should be in the 1 state indicated by the upper horizontal line in the figure, and the last 13 sets of fault data should be in the -1 state indicated by the next horizontal line, the red solid circle indicates the classification result of the data after LSSVM simulation., if the open circle of a certain group of data and the red solid circle coincide, it means that the 


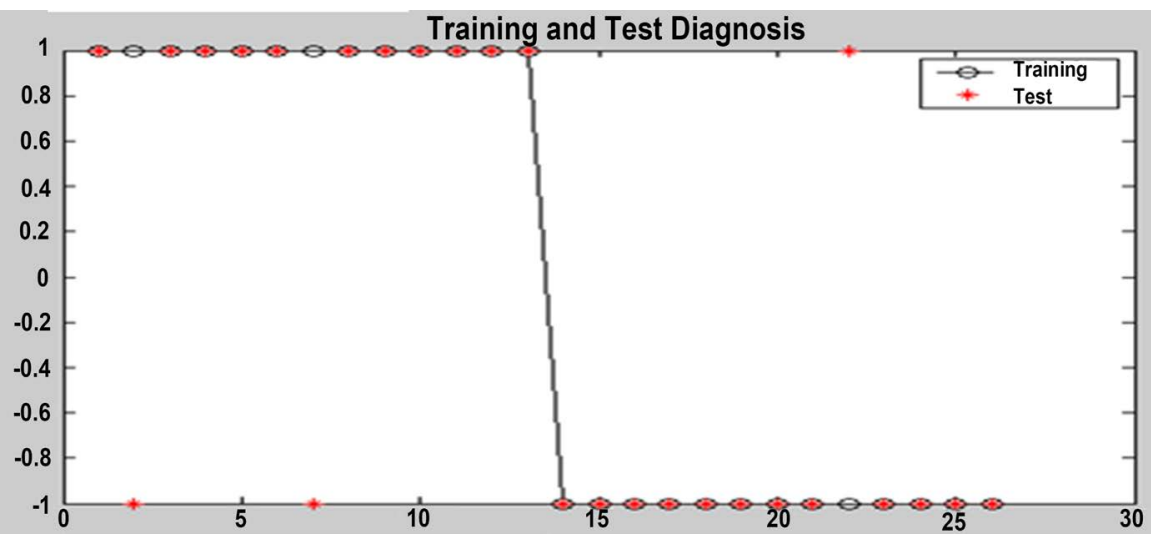

Figure 6. Simulating result in diagonal direction of definition 3.

simulation result of this set of data is correct, otherwise the error. Figure 6 shows that, in the definition of three modes, the feature vector obtained by the high-frequency is identified. There are only two groups in the normal state, and one group in the fault state has three groups of recognition errors, and the correct rate is over $85 \%$. For further analysis, the third-order cumulant of the real number is also subjected to the same experiment described above, and the results are also shown in Table 2. At the same time, according to the above method, when the oil pressure is $1 \mathrm{MPa}, 3 \mathrm{MPa}, 5 \mathrm{MPa}$, respectively, the absolute third-order cumulant of the two states is taken as an absolute value, and each group is selected as shown in Figure 7 and Figure 8. Its dimensions are similar to Figure 2 and Figure 3. It can be seen from Figure 7 and Figure 8 that, compared with the complex third-order cumulant, the real third-order cumulant between the fault state diagram and the normal state diagram, the degree of density of the peak distribution is more similar.

\section{Analysis of Experimental Results}

As mentioned earlier, the high-order cumulant can automatically suppress the influence of Gaussian background noise (colored or white), and the two-dimensional wavelet function decomposition can decompose the low-frequency part of the scale $\mathrm{j}$ into four parts. In this paper, the two-dimensional wavelet method is used to extract the features of the normal signal and the fault signal in the high frequency part in three different coupling modes, and use the extracted information for fault diagnosis.

The results show that, because the third-order cumulant contains different frequency information under different coupling modes, the correct rate obtained is also different. The experimental results are shown in Table 2.

\section{Conclusion}

Although the mechanism of various mechanical faults is different, each fault has the most essential difference from the normal state. For example, the fault in this paper is caused by adding a core to the pressure reducing valve in the normal 

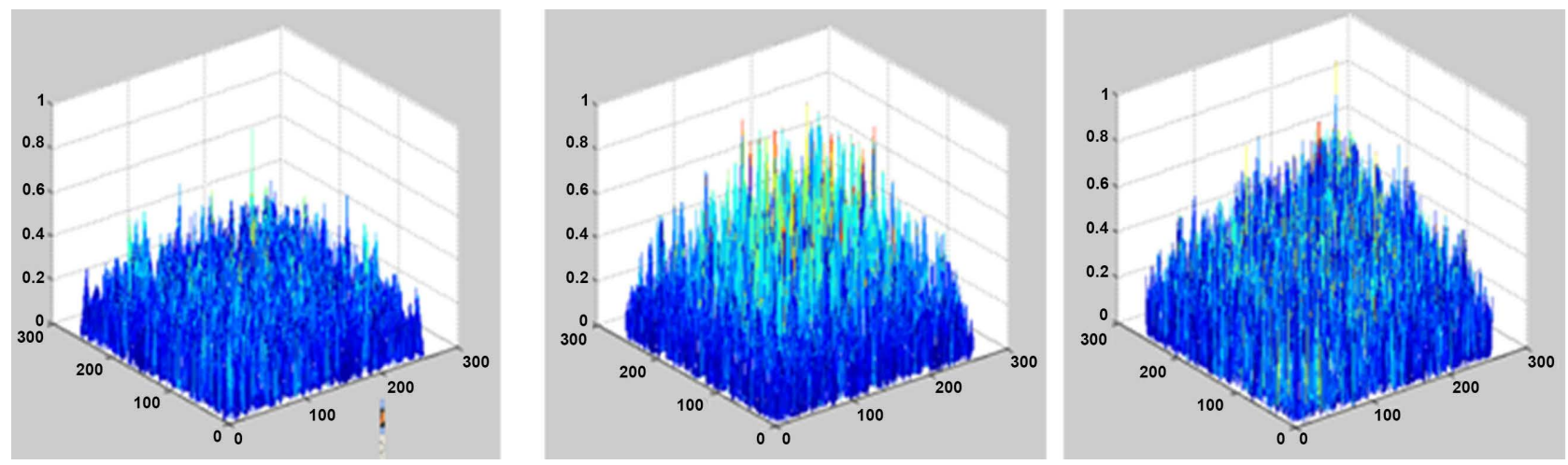

Figure 7. Real three order cumulants in normal state.
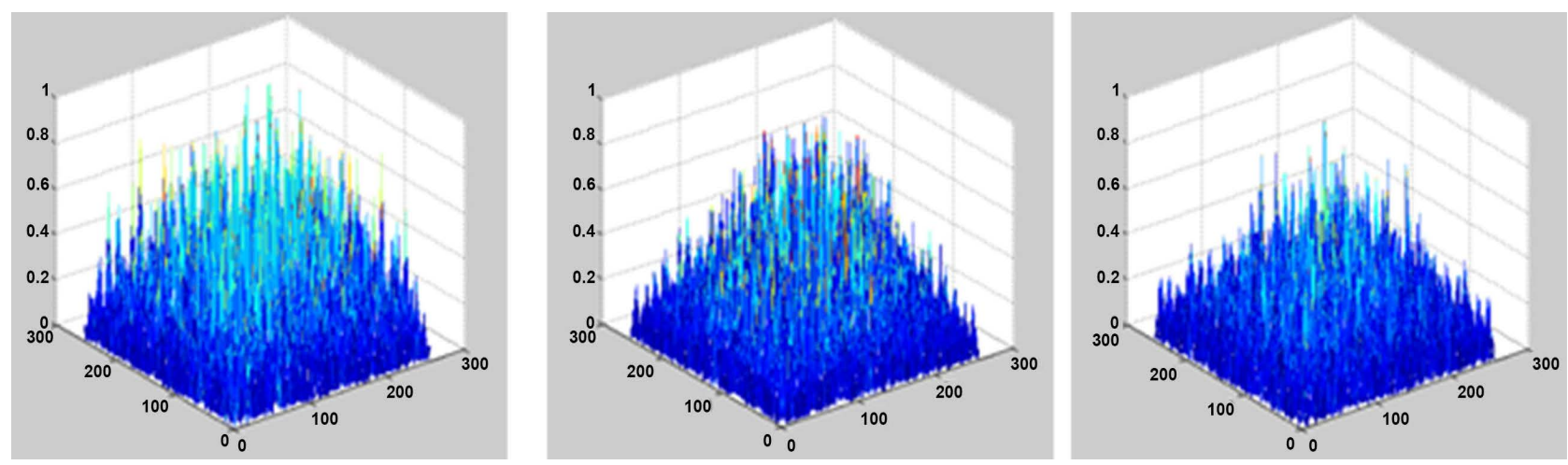

Figure 8. Real three order cumulants in fault state.

Table 2. Simulating error numbers in all kinds of definition.

\begin{tabular}{cc}
\hline mode & error numbers \\
\hline Definition 1 & 5 \\
Definition 2 & 7 \\
Definition 3 & 3 \\
real third-order cumulant & 6 \\
\hline
\end{tabular}

state. The essential difference is that the signal in the fault state is more likely to differ most in certain signal characteristics than the signal in the normal state. In this paper, through the different definitions of the complex third-order cumulant, the two-dimensional wavelet extracts the feature values in high frequency part to find the best diagnostic effect, and has achieved a certain degree of success.

\section{Acknowledgements}

This paper is supported by Research Foundation of the Nanchang Normal University for Doctors (NSBSJJ2018014). National Natural Science Foundation of China (61562063) Science and Technology Project of Jiangxi Provincial Education Department (GJJ171113). 


\section{Conflicts of Interest}

The author declares no conflicts of interest regarding the publication of this paper.

\section{References}

[1] Zhang, X.D. (2002) Modern Signal Processing. TsingHua University Press, Beijing, 263-281.

[2] Cai, Q.Z. and Huang, Y.J. (2009) Study on Autoregressive Trispectrum Slices. Chinese Journal of Scientific Instrument, 30, 345-350.

[3] Shao, R.P., Huang, X.N. and Liu, H.Y. (2008) Fault Detection and Diagnosis of Gear System Based on Higher Order Cumulants. Journal of Mechanical Engineering, 44, 161-168.

[4] Duan, X.Y., Wang, Y.S. and Su, Y.S. (2010) Application of Slice Bispectrum Analysis to Fault Diagnosis. Journal of Vibration, Measurement \& Diagnosis, 30, 581-584.

[5] Yang, D.M., Stronach, A.F., Macconnell, P., et al. (2002) Third Order Spectral Techniques for the Diagnosis of Motor Bearing Condition Using Artificial Neural Networks. Mechanical Systems \& Signal Processing, 16, 391-411. https://doi.org/10.1006/mssp.2001.1469

[6] Kocur, D. and Stanko, R. (2000) Order Bispectrum: A New Tool for Reciprocated Machine Condition Monitoring. Mechanical Systems \& Signal Processing, 14, 871-890. https://doi.org/10.1006/mssp.2000.1307

[7] Raughveer, M.R. (1990) Time-Domain Approaches to Quadratic Phase Coupling Estimation. IEEE Transactions on Automatic Control, 35, 48-56. https://doi.org/10.1109/9.45142

[8] Zhang, Y. and Wang, S.X. (1998) The Slice Spectra Approach to Nonlinear Phase Coupling Analysis. Acta Electronic Sinica, 26, 104-109.

[9] Li, Y.G., Liu, J. and Zhu, Q.B. (2005) Study of Orbit Analysis Based on Complex Process High Order Cumulant Spectrum. Journal of Mechanical Engineering, 41, 157-161.

[10] Wu, W.B. and Huang, Y.J. (2011) Fault Diagnosis of Reducing Valve Based on Bispectra Slices. Computer Measurement \& Control, No. 10, 2413-2416. (In Chinese) 